\title{
I-FiBH trial: intravenous fluids in benign headaches - a randomised, single-blinded clinical trial
}

\author{
Tony Zitek (1) , ${ }^{1}$ Tiffany Sigal, ${ }^{2}$ Gina Sun, ${ }^{3}$ Chris Martin Manuel, ${ }^{4}$ Khanhha $\operatorname{Tran}^{4}$
}

\begin{abstract}
Handling editor Ellen Weber
'Department of Emergency Medicine, Kendall Regional Medical Center, Miami, Florida, USA

${ }^{2}$ Department of Emergency Medicine, Mike O'Callaghan Federal Medical Center, Nellis Afb, Nevada, USA

${ }^{3}$ Department of Emergency Medicine, University of Nevada, Las Vegas School of Medicine, Las Vegas, Nevada, USA ${ }^{4}$ Department of Emergency Medicine, University Medical Center of Southern Nevada, Las Vegas, Nevada, USA
\end{abstract}

Correspondence to Dr Tony Zitek, Kendall Regional Medical Center, Miami, FL 33175, USA: zitek10@gmail.com

Received 20 December 2019 Revised 21 May 2020

Accepted 25 May 2020

Published Online First

3 July 2020

A Check for updates

(c) Author(s) (or their employer(s)) 2020. No commercial re-use. See rights and permissions. Published by BMJ.

To cite: Zitek T, Sigal T, Sun G, et al. Emerg Med J 2020:37:469-473.

\section{ABSTRACT}

Background Many emergency physicians use an intravenous fluid bolus as part of a 'cocktail' of therapies for patients with headache, but it is unclear if this is beneficial. The objective of this study was to determine if an intravenous fluid bolus helps reduce pain or improve other outcomes in patients who present to the ED with a benign headache.

Methods This was a randomised, single-blinded, clinical trial performed on patients aged 10-65 years old with benign headaches who presented to a single ED in Las Vegas, Nevada, from May 2017 to February 2019. All patients received prochlorperazine and diphenhydramine, and they were randomised to also receive either $20 \mathrm{~mL} /$ $\mathrm{kg}$ up to $1000 \mathrm{~mL}$ of normal saline (the fluid bolus group) or $5 \mathrm{~mL}$ of normal saline (the control group). The primary outcome was the difference between groups in mean pain reduction $60 \mathrm{~min}$ after the initiation of treatment. Secondarily, we compared groups with regards to pain reduction at $30 \mathrm{~min}$, nausea scores, the use of rescue medications and disposition.

Results We screened 67 patients for enrolment, and 58 consented. Of those, 35 were randomised to the fluid bolus group and 23 to the control group. The mean pain score dropped by $48.3 \mathrm{~mm}$ over $60 \mathrm{~min}$ in the fluid bolus group, compared with $48.7 \mathrm{~mm}$ in the control group. The between groups difference of $0.4 \mathrm{~mm}(95 \% \mathrm{Cl}$ -16.5 to 17.3 ) was not statistically significant $(p=0.96)$. Additionally, no statistically significant difference was found between groups for any secondary outcome. Conclusion Though our study lacked statistical power to detect small but clinically significant differences, ED patients who received an intravenous fluid bolus for their headache had similar improvements in pain and other outcomes compared with those who did not. Trial registration number NCT03185130.

\section{INTRODUCTION}

Headache was the fifth most common reason for patients to come to the ED in the USA in $2016 .^{1}$ As part of a 'cocktail' of therapies, an intravenous fluid bolus is given to patients with headaches in the ED about $40 \%$ of the time. ${ }^{2}$ While dehydration may precipitate a migraine, ${ }^{3}$ there are very little data to support the use of intravenous fluids for patients with primary headaches in the ED. Despite the lack of data on this subject, the use of intravenous fluids for headaches may have increased over the last 20 years. ${ }^{4}$

Until recently, there were no randomised trials assessing the efficacy of intravenous fluids for
Key messages

What is already known on this subject

- Although many physicians use an intravenous fluid bolus as part of their treatment for headaches, the benefit of doing so is unclear.

What this study adds

- Consistent with two other small randomised trials, this study found no evidence of benefit to the use of an intravenous fluid bolus for patients without severe dehydration who present to the ED with a headache. However, the study was underpowered to detect a small benefit of an intravenous fluid bolus.

patients with headaches. However, a study of 49 adult patients from a New Jersey ED published in 2019 found no improvement in headache pain scores in patients randomised to receive a $1 \mathrm{~L}$ intravenous fluid bolus compared with those randomised who received $10 \mathrm{~mL} /$ hour of fluid. ${ }^{5}$ Similarly, there is only one randomised trial on this topic for paediatric patients, and in that study of 45 paediatric patients, there was no statistically significant pain reduction for patients who received a $10 \mathrm{~mL} / \mathrm{kg}$ intravenous fluid bolus compared with those who did not. ${ }^{6}$

Given the frequency with which patients present to the ED with headaches and given the paucity of data on this subject, we performed a randomised trial to assess the effect of an intravenous fluid bolus on pain reduction in ED patients with headaches.

\section{METHODS}

\section{Study design and setting}

This was a single-centre, single-blinded, randomised controlled trial of patients presenting to the ED with a chief problem of headache. The hospital where data collection occurred is an academic, county facility in Las Vegas, Nevada. The ED at this facility is split into an adult ED and a paediatric ED. The adult ED has an annual census of approximately 80 000 , and the paediatric ED has an annual census of approximately 30000 .

\section{Patient and public involvement}

Patients were not involved in the production of this research study. 


\section{Selection of participants}

We enrolled a convenience sample of patients based on the availability of research assistants who were knowledgeable of the study protocol. Research assistants were generally in the ED from 14:00 to 22:00.

Patients were eligible for inclusion if they presented to the ED with a chief problem of headache, were between 10 and 65 years old and had a normal neurological examination. Patients were excluded if they were pregnant, had meningeal signs, had a fever, had suspected acute angle closure glaucoma, had head trauma or a lumbar puncture within the previous 2 weeks, had a thunderclap onset of their headache, had a known allergy to one of their study drugs, had a history of intracranial hypertension, were a prisoner, were non-English speaking (or had a non-English speaking guardian, if paediatric), had received analgesics or intravenous fluids prior to enrolment or were deemed to have severe dehydration (as determined by the treating physician's history and physical exam). These inclusion and exclusion criteria were designed to select for a group of patients with likely benign, primary headaches in whom there would not be a clear indication for intravenous fluids (except the headache).

Notably, we intentionally did not exclusively enrol patients with migraines based on the International Classification of Headache Disorders definition. ${ }^{7}$ This is because, in our experience, emergency physicians rarely use this definition to guide their treatment, and patients with primary headaches of all sorts tend to get similar treatments. Moreover, previous data have found that patients presenting to the ED with an undifferentiated headache improve with the same medications regardless of whether or not they meet the definition of migraine or another primary headache disorder. ${ }^{8-11}$

Patients who met all inclusion criteria and none of the exclusion criteria were approached about possible enrolment. Enrolled adult patients all signed written, informed consent. The guardians signed consent for paediatric patients, and the paediatric patients provided verbal assent.

\section{Study protocol}

After enrolment, all patients received standardised study medications, and they were randomised to the fluid bolus group or the control group. The standardised medications were prochlorperazine $0.15 \mathrm{mg} / \mathrm{kg}$ up to $10 \mathrm{mg}$ intravenous and diphenhydramine $1 \mathrm{mg} / \mathrm{kg}$ up to $50 \mathrm{mg}$ intravenous. The diphenhydramine was administered first, immediately followed by prochlorperazine. Prochlorperazine was chosen for this study because it is one of the most effective medications for acute headaches, with data suggesting that it is superior to ketorolac, ${ }^{12}$ sumatriptan, ${ }^{13}$ sodium valproate, ${ }^{14}$ promethazine, ${ }^{15}$ hydromorphone ${ }^{16}$ and ketamine. ${ }^{17}$ The efficacy of prochlorperazine is similar to that of metoclopramide. ${ }^{18} 19$ The use of diphenhydramine in combination with prochlorperazine is supported by some evidence that suggests it may prevent akathisia, ${ }^{20}$ and it is used in this fashion in our ED. Therefore, we used diphenhydramine with prochlorperazine as described above.

After providing informed consent, patients underwent simple randomisation to either the fluid bolus group or the control group. The treating physician consulted a folder that contained a list specifying the group assignment. Each row of the list corresponded to a patient, and was covered by a small slip of opaque paper that was removed at the time of enrolment to reveal the group assignment. This list was constructed at the beginning of the study using a random number generator by a research staff member not involved in data collection. The list was designed to lead to equal sized groups at the conclusion of the study.

Patients randomised to the fluid bolus group received $20 \mathrm{~mL} /$ $\mathrm{kg}$ up to $1000 \mathrm{~mL}$ of normal saline over 1 hour while those in the control group received normal saline at $5 \mathrm{~mL} /$ hour. To ensure the intravenous fluids were given over 1 hour, the fluids were given using a pump. The intravenous bag and pump were covered with an opaque sheet so that the patient and research assistant performing outcome assessments were blinded from how much fluid the patient received. The treating physician and nurse were not blinded to the amount of fluids administered. Physicians were instructed not to administer any additional headache medications ('rescue medications') for at least $30 \mathrm{~min}$.

Just prior to the initiation of treatment, under the monitoring of a research assistant, enrolled patients filled out a data collection form that included age, sex, race and baseline headache and nausea severity scores using a $100 \mathrm{~mm}$ visual analogue scale (VAS). After the administration of the study medications, the research assistant left the patient's bedside, and returned 30 and $60 \mathrm{~min}$ later to obtain repeat headache and nausea severity scores. VAS pain and nausea scores have previously been validated to assess pain and nausea in the ED, ${ }^{22}$ and a difference of $13 \mathrm{~mm}$ between groups on the $100 \mathrm{~mm}$ pain VAS pain scale is considered to be clinically important. ${ }^{21}$

The presence or absence of vomiting was also assessed at baseline, $30 \mathrm{~min}$ and $60 \mathrm{~min}$. The need for rescue medications and disposition was abstracted from the patient's chart at a later time. Trained research assistants called the patients $24-48$ hours post discharge by phone to ask if they were having a headache at that time (yes or no). If the patient was admitted, the patient was approached in the hospital by research staff for follow-up. As is typical with clinical trials, patients were monitored for unexpected adverse events and serious adverse events. Given the safety from a small intravenous fluid bolus, no other specific adverse events were tracked.

\section{Outcome measures}

The primary outcome measure was the difference in mean pain score improvement between the fluid bolus group and the control group at $60 \mathrm{~min}$. Secondarily, we measured the differences between the groups with regards to improvements in pain scores at $30 \mathrm{~min}$ and improvements in nausea scores at 30 and $60 \mathrm{~min}$. Additionally, rates of vomiting, use of rescue medications, rates of admission and headache resolution $24-48$ hours after treatment were assessed.

\section{Data analysis}

As above, a difference of $13 \mathrm{~mm}$ between groups suggests a clinically important difference in pain. ${ }^{21}$ Therefore, assuming a SD of 22 (based on prior data from our institution ${ }^{17}$ ), we calculated that we would need 45 patients in each group to find a clinically important difference in pain score reduction between groups at 60 min with at least $80 \%$ power and an alpha of 0.05 .

The individual VAS measurements were first compared using repeated measures analysis of variance tests without controlling for any baseline characteristics. Subsequently, repeated measures analysis of variance tests were performed using gender, ethnicity and mean initial nausea scores as control variables (as there were non-statistically significant, moderate-sized differences between groups for these variables). No substantial differences were noted in results of the latter analysis as compared with the former, and so the results of the simpler former analysis are reported below. Model fit diagnostics were assessed, and the models were found 
Figure 1: Demonstrates patient flow.

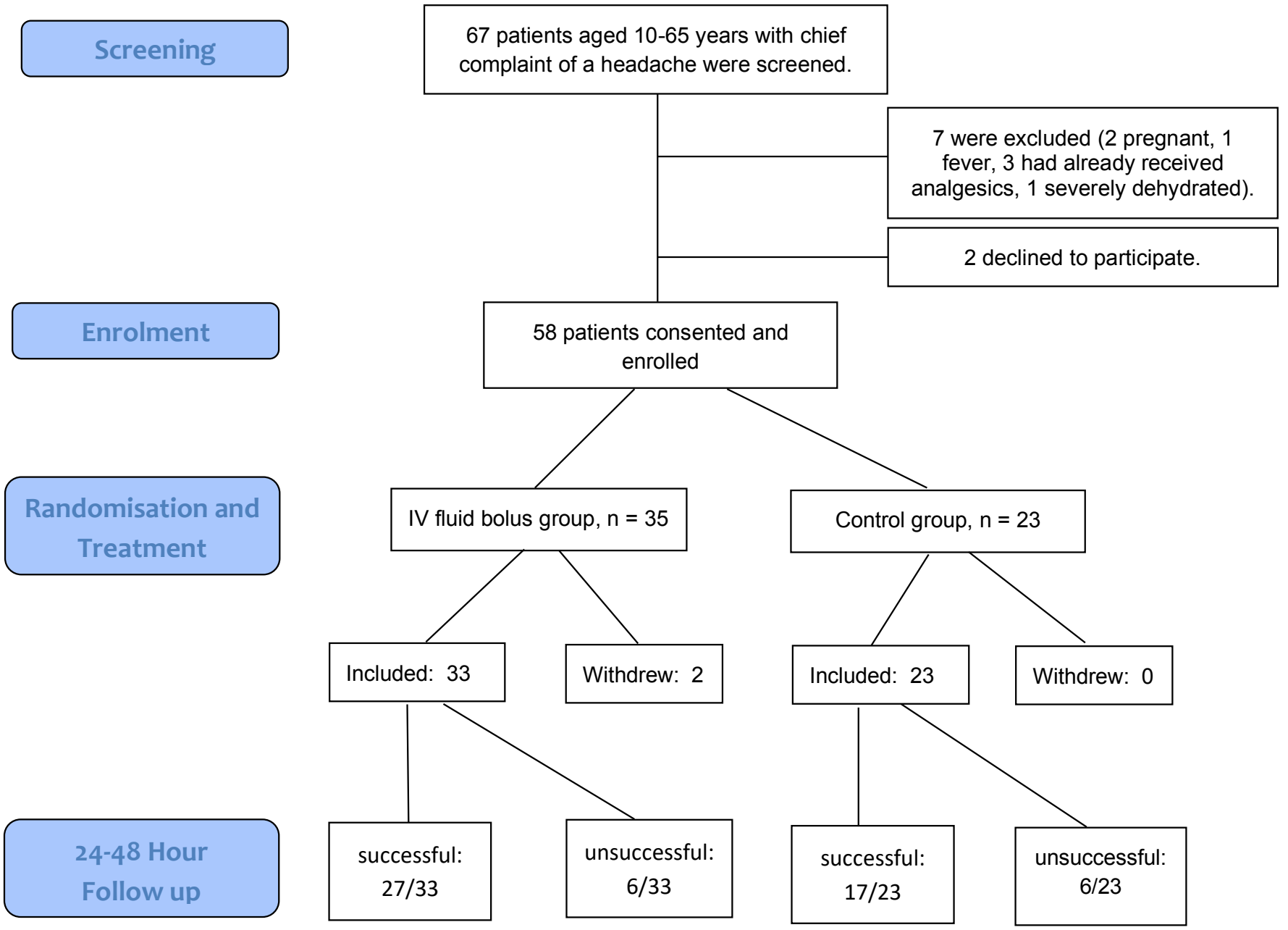

Figure 1 Demonstrates patient flow. IV, intravenous.

to be adequate. Other continuous variables were compared between groups using t-tests for normally distributed data and Mann-Whitney U tests for non-normal data. $\chi^{2}$ tests or Fisher's exact tests (for event counts less than 5) were used to compare categorical variables.

\section{RESULTS}

Between May 2017 and February 2019, 67 patients were screened, and 58 were enrolled. Unfortunately, after one of the investigators left the facility where data collection occurred, enrolment slowed. Consequently, the study was stopped before reaching its enrolment goal of 90 . Of the 58 patients enrolled, 35 were randomised to the fluid bolus group and 23 to the control group. Figure 1 demonstrates the details of patient flow. Two of the 58 patients enrolled (both in the fluid bolus group) decided they did not want to stay for a full $60 \mathrm{~min}$ after treatment, so they withdrew from the study after the 30 min measurements. This left 56 patients for calculation of the primary outcome. No patients who were randomised to the control group received a fluid bolus. All patients in the fluid bolus group received the full fluid bolus with the exception of the two patients who withdrew from the study early (stating they did not want to wait a full hour after the initiation of treatment without further explanation); each of them received about half the fluid bolus.
Enrolled patients ranged from 10 to 65 years old (median 29.5 ), and were $63.8 \%$ female. Table 1 shows the baseline characteristics of both groups; there were no statistically significant differences between groups. Among the 58 enrolled patients, the primary final diagnosis for all patients was some form of headache: 3 were diagnosed with tension headaches, 8 with

\begin{tabular}{|c|c|c|}
\hline Characteristic* & $\begin{array}{l}\text { Fluid bolus group, } \\
\mathrm{n}=35\end{array}$ & Control group, $n=23$ \\
\hline$\%$ Female & 57.1 & 73.9 \\
\hline Mean age (years) & $32.3 \pm 14.1$ & $31.7 \pm 14.7$ \\
\hline \multicolumn{3}{|l|}{ Ethnicity } \\
\hline$\%$ Hispanic & 34.3 & 21.7 \\
\hline \multicolumn{3}{|l|}{ Race } \\
\hline$\%$ White & 54.3 & 60.9 \\
\hline$\%$ Black & 40.0 & 34.8 \\
\hline Mean initial pain score $(0-100)$ & $72.3 \pm 23.8$ & $71.5 \pm 26.3$ \\
\hline Mean initial nausea score $(0-100)$ & $32.2 \pm 37.8$ & $42.9 \pm 34.6$ \\
\hline$\%$ Vomiting with headache & 31.4 & 30.4 \\
\hline
\end{tabular}


Table 2 A comparison of various outcome measures between groups

\begin{tabular}{|c|c|c|c|c|}
\hline & Fluid bolus group* $(n=35)$ & Control group* $(n=23)$ & Difference $(95 \% \mathrm{Cl})$ & $P$ value \\
\hline Mean pain score at $0 \mathrm{~min}$ & $72.3 \pm 23.8$ & $71.5 \pm 26.3$ & $0.8(-13.3$ to 14.9$)$ & 0.91 \\
\hline Mean pain score at $30 \mathrm{~min}$ & $40.5 \pm 33.1$ & $36.7 \pm 31.1$ & $3.8(-14.0$ to 21.5$)$ & 0.66 \\
\hline Mean pain reduction at $30 \mathrm{~min}$ & $31.8 \pm 27.1$ & $34.8 \pm 29.6$ & $3.0(-13.0$ to 19.0$)$ & 0.70 \\
\hline Mean pain score at $60 \mathrm{~min}$ & $24.3 \pm 32.4$ & $22.8 \pm 30.3$ & $1.5(-16.0$ to 19.1$)$ & 0.86 \\
\hline Mean pain reduction at $60 \mathrm{~min}$ & $48.3 \pm 31.0$ & $48.7 \pm 29.3$ & $0.4(-16.5$ to 17.3$)$ & 0.96 \\
\hline Mean nausea score at $0 \mathrm{~min}$ & $32.2 \pm 37.8$ & $42.9 \pm 34.6$ & $10.7(-9.3$ to 30.6$)$ & 0.28 \\
\hline Mean nausea score at $30 \mathrm{~min}$ & $13.5 \pm 26.7$ & $16.3 \pm 20.8$ & $2.8(-10.3$ to 15.7$)$ & 0.67 \\
\hline Mean nausea reduction at $30 \mathrm{~min}$ & $18.7 \pm 31.4$ & $26.6 \pm 33.2$ & $7.9(-10.2$ to 26.0$)$ & 0.38 \\
\hline Mean nausea score at $60 \mathrm{~min}$ & $7.1 \pm 19.4$ & $11.1 \pm 18.2$ & $4.0(-6.5$ to 14.5$)$ & 0.44 \\
\hline Mean nausea reduction at $60 \mathrm{~min}$ & $25.2 \pm 34.2$ & $31.8 \pm 33.4$ & $6.6(-12.4$ to 25.6$)$ & 0.48 \\
\hline Vomited after treatment & 0 & 0 & N/A & $\mathrm{N} / \mathrm{A}$ \\
\hline$\%$ Receiving rescue medications $\dagger$ & 20.0 & 13.0 & $7.0(-12.2$ to 26.1$)$ & 0.49 \\
\hline$\%$ Admitted & 5.7 & 0 & $5.7(-2.0$ to 13.4$)$ & 0.24 \\
\hline$\%$ With a headache at $24-48$ hours & $40.7(11 / 27)$ & $23.5(4 / 17)$ & $17.2(-10.2$ to 44.6$)$ & 0.24 \\
\hline
\end{tabular}

*Plus-minus values are \pm SD

tRescue medications included morphine, hydromorphone, ketorolac, magnesium, rizatriptan, diazepam, lorazepam, haloperidol, acetaminophen and dexamethasone. N/A, Not applicable.

migraines and the remaining 47 were diagnosed with (nonspecific) headache.

Regarding the primary outcome, over $60 \mathrm{~min}$, the mean pain score in the fluid bolus group dropped by $48.3 \mathrm{~mm}$, while it dropped by $48.7 \mathrm{~mm}$ in the control group. The difference in mean pain score reduction over $60 \mathrm{~min}$ between groups of $0.4 \mathrm{~mm}(95 \% \mathrm{CI}-16.5$ to $17.3 \mathrm{~mm})$ is not statistically significant $(\mathrm{p}=0.96)$, nor would it be clinically significant.

Given that two patients in the fluid bolus group left prior to $60 \mathrm{~min}$, a sensitivity analysis was performed assuming the patients left because their pain completely resolved. With this assumption, the reduction in mean pain score increases to $49.4 \mathrm{~mm}$ in the fluid bolus group. The between groups difference of $0.7 \mathrm{~mm}$ (95\% CI -16.0 to $17.3 \mathrm{~mm}$ ) now slightly favours the fluid bolus group but is still neither statistically significant $(p=0.94)$ nor clinically significant.

Regarding secondary outcomes, there were no statistically significant differences between groups with regards to mean pain score reductions at $30 \mathrm{~min}$, mean nausea score reductions at 30 or $60 \mathrm{~min}$, the percentage who received rescue medications, the percentage who were admitted or the percentage of patients who had a headache at 24-48 hour follow-up. Although a little over $30 \%$ of patients had vomited prior to enrolment in each group, no patients vomited during data collection in either group. No unexpected adverse events or serious adverse events occurred in either group. See table 2 for a detailed assessment of all outcomes.

Of note, two patients were admitted to the hospital in the fluid bolus group, while none were admitted in the control group. One of the two admitted patients was admitted for intractable headache. The second patient who was admitted had marked hypertension with his headache. The emergency physician did not order any diagnostic tests and treated the patient per study protocol. However, the patient's primary care doctor was concerned, and asked the patient be admitted for a possible hypertensive emergency. This patient eventually had a CT scan of the brain and blood tests done. None of these tests identified any explanation for the patient's headache, and the patient was discharged from the hospital the next day.

\section{DISCUSSION}

This is the third randomised trial assessing the effects of an intravenous fluid bolus on pain scores in patients presenting to the ED with headaches. ${ }^{56}$ While our study was small, it is nevertheless the largest randomised trial about intravenous fluid boluses for headaches to date. Our study was performed contemporaneously with the one by Jones et $a l,{ }^{5}$ and the studies were quite similar. Both were randomised controlled trials comparing an intravenous fluid bolus to a minimal amount of intravenous fluids for patients with headaches in the ED. Just as our trial (which included both adult and paediatric patients) found no improvement in pain or nausea scores for patients receiving a fluid bolus compared with the control group, the study by Jones et al (which included 49 adult patients with migraines) and the study by Richer et al (which included 45 paediatric patients with migraines) found no evidence of benefit to the administration of a fluid bolus. ${ }^{56}$ Additionally, a post hoc analysis from another clinical trial concluded that intravenous fluids did not improve pain outcomes in patients receiving metoclopramide. ${ }^{23}$

Thus, while dehydration may be associated with headaches, ${ }^{324}$ and some dehydrated patients may benefit from an intravenous fluid bolus, there is now increasing evidence that a fluid bolus should not be a routine part of the ED cocktail for primary headaches. There is, in fact, not even a signal of benefit in the data for intravenous fluid boluses for headaches. Although there were no statistically significant differences, the three prior studies on this topic ${ }^{5623}$ and ours all found less pain reduction in the fluid bolus group.

While a $1 \mathrm{~L}$ intravenous fluid bolus is unlikely to cause serious harm to patients without volume-sensitive conditions like severe heart failure, it is noteworthy that there were trends towards harm in this study. Indeed, while not statistically significant, our study found that patients in the fluid bolus group received more rescue medications, be admitted and have a headache 24-48 hours after discharge as compared with the control group. When considering the lack of proven benefit, the possibility of harm and the expense of a $1 \mathrm{~L}$ bag of normal saline (which costs US\$713 for a patient at one of the investigators' affiliated institutions ${ }^{25}$ ), the routine use of an intravenous fluid bolus for primary headaches should be questioned. 
However, when interpreting the results of this study, there are several limitations to consider. First of all, we enrolled a convenience sample of patients at a single hospital, and our results may have been affected by volunteer and selection bias. It is thus possible that our results are not generalisable to other populations. Second, our study was single blinded (not double blinded), so it is possible that the physicians' or nurses' knowledge of the patient's randomisation may have biased the results. Furthermore, with regards to blinding, our strategy to blind the patients and outcome assessors was not foolproof; some unblinding may have occurred which also could have biased the results. However, the study by Jones et al used a similar method of blinding and found it to be effective. ${ }^{5}$

Perhaps the most important limitation of the study was that it was underenrolled, and as a result of the means by which randomisation was performed, the underenrolment resulted in uneven group numbers. Consequently, there may be a small beneficial effect to an intravenous fluid bolus that was not detected in this study. That being said, when considering the results of all three randomised trials on this subject, this seems relatively unlikely.

One final limitation to consider is that our study and previous publications ${ }^{5623}$ have assessed the efficacy of an intravenous fluid bolus for patients receiving dopamine antagonists (either metoclopramide or prochlorperazine) for their headache. It is possible that an intravenous fluid bolus would have a more positive effect if given with a different class of medications or without any medications. However, dopamine antagonists are the recommended first-line headache medications at this time, ${ }^{26}$ so it would not be fruitful to assess the efficacy of an intravenous fluid bolus on pain in patients receiving other types of medications or no medications.

In conclusion, our study lacked statistical power to detect small differences between groups, but we found no evidence of benefit to administering an intravenous fluid bolus to a group of patients without severe dehydration who presented to the ED with benign headaches. We recommend against the routine use of an intravenous fluid bolus as part of an ED headache cocktail.

Contributorship Statement TZ contributed by designing the study, performing data analysis, drafting the manuscript and providing final approval for the manuscript to be sent in for publication. TS contributed by formulating the research question and hypothesis; she also helped with data collection. GS, CMM and KT helped in data collection and data analysis. All authors helped revise the manuscript.

Funding The authors have not declared a specific grant for this research from any funding agency in the public, commercial or not-for-profit sectors.

Competing interests None declared.

Patient consent for publication Not required.

Ethics approval This study was approved by the University Medical Center of Southern Nevada's institutional review board (number: UMC-2017-54).

Provenance and peer review Not commissioned; externally peer reviewed.

Data availability statement Data are available upon reasonable request. Deidentified participant data are available upon request via https://orcid.org/00000002-4357-6611.

ORCID iD

Tony Zitek http://orcid.org/0000-0002-4357-6611

\section{REFERENCES}

1 National Hospital ambulatory medical care survey: 2016 emergency department summary tables. Available: https://www.cdc.gov/nchs/data/nhamcs/web_tables/ 2016_ed_web_tables.pdf [Accessed 12 Jul 2019].

2 Jones CW, Gaughan JP, McLean SA. Epidemiology of intravenous fluid use for headache treatment: findings from the National Hospital ambulatory medical care survey. Am J Emerg Med 2017;35:778-81.

3 Blau JN. Water deprivation: a new migraine precipitant. Headache 2005;45:757-9.

4 Ruzek M, Richman P, Eskin B, et al. Ed treatment of migraine patients has changed. Am J Emerg Med 2019;37:1069-72.

5 Jones CW, Remboski LB, Freeze B, et al. Intravenous fluid for the treatment of emergency department patients with migraine headache: a randomized controlled trial. Ann Emerg Med 2019;73:150-6.

6 Richer L, Craig W, Rowe B. Randomized controlled trial of treatment expectation and intravenous fluid in pediatric migraine. Headache 2014;54:1496-505.

7 Headache Classification Committee of the International Headache Society (IHS). The International classification of headache disorders, 3rd edition (beta version). Cephalalgia 2013;33:629-808.

8 Jones J, Sklar D, Dougherty J, et al. Randomized double-blind trial of intravenous prochlorperazine for the treatment of acute headache. JAMA 1989;261:1174-6.

9 Miner JR, Fish SJ, Smith SW, et al. Droperidol vs. prochlorperazine for benign headaches in the emergency department. Acad Emerg Med 2001;8:873-9.

10 Friedman BW, Adewunmi V, Campbell C, et al. A randomized trial of intravenous ketorolac versus intravenous metoclopramide plus diphenhydramine for tensiontype and all nonmigraine, noncluster recurrent headaches. Ann Emerg Med 2013;62:311-8.

11 Cicek M, Karcioglu 0, Parlak I, et al. Prospective, randomised, double blind, controlled comparison of metoclopramide and pethidine in the emergency treatment of acute primary vascular and tension type headache episodes. Emerg Med J 2004;21:323-6.

12 Seim MB, March JA, Dunn KA. Intravenous ketorolac vs intravenous prochlorperazine for the treatment of migraine headaches. Acad Emerg Med 1998;5:573-6.

13 Kostic MA, Gutierrez FJ, Rieg TS, et al. A prospective, randomized trial of intravenous prochlorperazine versus subcutaneous sumatriptan in acute migraine therapy in the emergency department. Ann Emerg Med 2010;56:1-6.

14 Tanen DA, Miller S, French T, et al. Intravenous sodium valproate versus prochlorperazine for the emergency department treatment of acute migraine headaches: a prospective, randomized, double-blind trial. Ann Emerg Med 2003;41:847-53.

15 Callan JE, Kostic MA, Bachrach EA, et al. Prochlorperazine vs. promethazine for headache treatment in the emergency department: a randomized controlled trial. J Emerg Med 2008;35:247-53.

16 Friedman BW, Irizarry E, Solorzano C, et al. Randomized study of IV prochlorperazine plus diphenhydramine vs IV hydromorphone for migraine. Neurology 2017:89:2075-82

17 Zitek T, Gates M, Pitotti C, et al. A Comparison of Headache Treatment in the Emergency Department: Prochlorperazine Versus Ketamine. Ann Emerg Med 2018:71:369-77.

18 Friedman BW, Esses D, Solorzano C, et al. A randomized controlled trial of prochlorperazine versus metoclopramide for treatment of acute migraine. Ann Emerg Med 2008;52:399-406.

19 Coppola M, Yealy DM, Leibold RA. Randomized, placebo-controlled evaluation of prochlorperazine versus metoclopramide for emergency department treatment of migraine headache. Ann Emerg Med 1995;26:541-6.

20 Vinson DR, Drotts DL. Diphenhydramine for the prevention of akathisia induced by prochlorperazine: a randomized, controlled trial. Ann Emerg Med 2001;37:125-31.

21 Gallagher EJ, Liebman M, Bijur PE. Prospective validation of clinically important changes in pain severity measured on a visual analog scale. Ann Emerg Med 2001;38:633-8.

22 Meek R, Kelly A-M, Hu XF. Use of the visual analog scale to rate and monitor severity of nausea in the emergency department. Acad Emerg Med 2009;16:1304-10.

23 Balbin JEB, Nerenberg R, Baratloo A, et al. Intravenous fluids for migraine: a post hoc analysis of clinical trial data. Am J Emerg Med 2016;34:713-6.

24 Blau JN, Kell CA, Sperling JM. Water-deprivation headache: a new headache with two variants. Headache 2004;44:79-83.

25 Detailed price list. Available: https://core.secure.ehc.com/src/util/detail-price-list/ EastFloridaDivision_KendallRegionalMedicalCenter_CM.csv [Accessed 24 Feb 2020].

26 Long BJ, Koyfman A. Benign headache management in the emergency department. J Emerg Med 2018;54:458-68. 